\title{
Fueling-controlled growth of massive black holes
}

\author{
Andrés Escala \\ Kavli Institute for Particle Astrophysics and Cosmology, \\ Stanford University / SLAC, 2575 Sand Hill Rd. MS 29, Menlo Park, CA 94025, USA. \\ email: andres@slac.stanford.edu
}

\begin{abstract}
We study the relation between nuclear massive black holes and their host spheroid gravitational potential. Using AMR numerical simulations, we analyze how gas is transported into the nuclear (central kpc) regions of galaxies. We study gas fueling onto the inner accretion disk (sub-pc scale) and star formation in a massive nuclear disk like those generally found in proto-spheroids (ULIRGs, SCUBA Galaxies). These sub-pc resolution simulations of gas fueling, which is mainly depleted by star formation, naturally satisfy the ' $\mathrm{M}_{\mathrm{BH}}-\mathrm{M}_{\mathrm{virial}}$ ' relation, with a scatter considerably less than that observed. We find that a generalized version of the Kennicutt-Schmidt Law for starbursts is satisfied, in which the total gas depletion rate $\left(\dot{\mathrm{M}}_{\mathrm{gas}}=\right.$ $\left.\dot{\mathrm{M}}_{\mathrm{BH}}+\dot{\mathrm{M}}_{\mathrm{SF}}\right)$ scales as $\mathrm{M}_{\mathrm{gas}} / \mathrm{t}_{\text {orbital }}$.
\end{abstract}

Keywords. quasars: general - galaxies: formation - black hole physics

\section{Introduction}

In the past years, it has been found that most nearby massive spheroids (elliptical and spiral bulges) host nuclear massive black holes (MBH) (Kormendy \& Richstone 1995), whose masses correlate with their host spheroid properties. Two correlations arise as the more relevant links between $\mathrm{MBH}$ and their hosts. First, the black hole mass correlates with host mass ('M $\mathrm{M}_{\mathrm{bh}}-\mathrm{M}_{\text {bulge }}$ ' relation; Marconi \& Hunt 2003; Haring \& Rix 2004). Second, their masses correlate with the average random velocities of the stars in their host (' $\mathrm{M}_{\mathrm{bh}}-\sigma$ ' relation; Ferrarrese \& Merritt 2000; Gebhardt et al. 2000).

The study of $\mathrm{MBH}$ growth by gas accretion is usually focused on the study of accretion disks. However, these accretion disks are Keplerian by nature and therefore have negligible masses compared to that of the $\mathrm{MBH}$. They must be continuously replenished, otherwise the mass of the $\mathrm{MBH}$ will not have considerable growth. The key question in the growth of MBHs by accretion is how to remove the large angular momentum of gas in a galaxy in order to funnel it into the accretion disk in the central sub-pc region -the so called 'Fueling Problem'-.

There are several mechanisms for fueling gas into kpc scales, such as galaxy mergers/interactions, bars and resonances (see Shlosman, Begelman \& Frank 1990 for a review). Gravitational torques in galaxy mergers arise as the dominant process for transporting large amounts of gas down to the central few hundred parsecs and for triggering most of the $\mathrm{MBH}$ growth. In a merger, after a violently relaxed core is formed at the center, most of the gas will settle in a nuclear disk (typically several hundred parsecs in diameter) that is rotationally supported against the overall (gas + stars) gravitational potential (Barnes 2002; Kazantzidis et al. 2005; Mayer et al. 2007). The MBHs will migrate to the center, and merge on a timescale relatively short compared to the lifetime of the nuclear disk (Escala et al. 2004, 2005; Dotti et al. 2006). In this paper, this massive nuclear disk, along with a central $\mathrm{MBH}$, is the starting point of our study. We focus on 
the mass fueling onto the inner accretion disk around a $\mathrm{MBH}$ (from the few hundred pc down to sub-pc scale).

\section{Numerical Setup}

The model consists of a massive gaseous disk surrounding a $\mathrm{MBH}$, both of which are embedded in a stellar spheroid. The spheroid is modeled by a time-independent external potential. We use the gravitational potential of an isothermal sphere for $\mathrm{r} \geqslant 100 \mathrm{pc}$, and the potential of a homogenous sphere for $\mathrm{r}<100 \mathrm{pc}$. We solve the hydrodynamic and Poisson equations using the ENZO adaptative mesh refinement hydrodynamics code (Bryan and Norman 1997). The adaptative cartesian grid covers a $1 \mathrm{kpc}^{3}$ region around the galactic center, with a spatial resolution of 0.24 pc. Radiative gas losses from an optically thin plasma are modeled with a cooling curve down to temperatures of $10^{4} \mathrm{~K}$. We use outflow (zero gradient) boundary conditions and in our simulations we use the following units: $\left[\right.$ Mass] $=1.76 \times 10^{10} \mathrm{M}_{\text {sun }},\left[\right.$ Velocity] $=276 \mathrm{kms}^{-1}$, and [Distance] $=1$ kpc. For modeling star formation, the criteria were used to decide whether a grid cell would produce a star follows th one in Cen \& Ostriker (1992). The massive black hole and its accretion disk are modeled as a sink particle that is initially placed at the center of the system.

The initial condition is a rotationally supported disk with a $1 / \mathrm{r}$ density profile, with a diameter of $600 \mathrm{pc}$ and a thickness of $30 \mathrm{pc}$. The total mass of the disk is $\mathrm{M}_{\mathrm{gas}}=$ $3 \times 10^{8} \mathrm{M}_{\text {sun }}$ and the initial mass of the central black hole is $\mathrm{M}_{\mathrm{BH}}=10^{6} \mathrm{M}_{\text {sun }}$. The initial temperature is set to $10^{4} \mathrm{~K}$ over the whole region. Random density and temperature fluctuations of less than $1 \%$ are added to the initial uniform disk. We performed six different runs in which we varied the mass of the bulge in such a way that the velocity dispersion of the bulge, $\sigma$, is: $110,163,216,270,321$ and $357 \mathrm{~km} \mathrm{~s}^{-1}$.

\section{Results}

In a previous work, Escala (2006 -hereafter E06-), we analyzed how gas is expected to be transported into the nuclear regions of galaxies using simple analytical models. E06 showed that the average mass fueling onto the inner accretion disk, $\langle\dot{M}\rangle$, is proportional to $\sigma^{3}$ (eq. 3 in E06). This result is valid for the different possible types of massive nuclear disks, and also for the different mechanisms of angular momentum transport, the zero-point being the only way to discriminate between them (see $\S 5$ in the same paper). E06 also coupled this result with the expected gas lifetime given by the Kennicutt-Schmidt Law (Kennicutt 1998; $\mathrm{t}_{\mathrm{gas}} \approx \mathrm{t}_{\mathrm{SF}}=\Sigma_{\text {gas }} / \dot{\Sigma}_{\mathrm{SF}} \propto \mathrm{t}_{\mathrm{orb}}=\mathrm{R}_{\mathrm{d}} / \sigma$ ), leading naturally to the ' $\mathrm{M}_{\mathrm{BH}}-\mathrm{M}_{\text {virial }}$ ' relation:

$$
\mathrm{M}_{\mathrm{BH}}=\dot{\mathrm{M}} \mathrm{t}_{\text {gas }} \propto \mathrm{R}_{\mathrm{d}} \sigma^{2} \propto \mathrm{M}_{\mathrm{virial}} .
$$

In this paper, we verify whether each of these conditions are satisfied in our simulations (see Escala 2007 for more details). We start by investigating whether the star formation rate in our model satisfies the Kennicutt-Schmidt Law: $\dot{\Sigma}_{\mathrm{SF}} / \Sigma_{\text {gas }}=\dot{\mathrm{M}}_{\mathrm{SF}} / \mathrm{M}_{\mathrm{gas}} \propto \mathrm{t}_{\mathrm{orb}}^{-1}$. Figure 1a shows the average gas depletion rate, $<\dot{M}_{\text {gas }}>$, against the orbital time $\left(=2 \pi \mathrm{R}_{\mathrm{d}} / \sigma\right)$ for the six different runs. Since the total gas mass $\mathrm{M}_{\mathrm{gas}}$ is the same for all the runs, the Kennicutt-Schmidt Law predicts that $<\dot{M}_{\text {gas }}>$ must be proportional to $1 / t_{\text {orb }}$, as indicated by the solid line in Fig. 1a. The open circles are the gas depletion rate including only the gas depleted by star formation described by in the KennicuttSchmidt Law. The filled circles are again the gas depletion rate for each simulation, but now including the gas depleted by star formation and by accretion onto the central black 
hole. The figure clearly shows that it is the total gas depletion rate that correlates best with the inverse of the orbital time $t_{\text {orb }}$, and not the gas depletion rate due to star formation only.

Kennicutt (1998) estimates the star formation rate by using the far infrared luminosity ( $\left.\mathrm{L}_{\mathrm{FIR}}\right)$. It is well known that $\mathrm{L}_{\mathrm{FIR}}$ has a relevant AGN contribution (approximately 1/3), so that what Kennicutt (1998) identifies as a star formation rate is in fact closer to the total gas depletion rate, as shown in our simulations. E06 also neglect the bh's mass accretion rate in the gas depletion timescale, making $\mathrm{t}_{\mathrm{gas}} \approx \mathrm{t}_{\mathrm{SF}} \propto \mathrm{t}_{\mathrm{orb}}$. Our simulations show that such an approximation is no longer needed, because it is the total (accretion plus star formation) gas depletion timescale itself that scales with the orbital time.

The second condition needed to be satisfied by our simulations is that $\langle\dot{M}\rangle$ is proportional to $\sigma^{3}$, something that was already tested in E06 for an adiabatic disk. We estimated $<\dot{M}>$ by the mass accretion rate onto the sink particle per orbital time $M_{\mathrm{BH}} / \mathrm{t}_{\mathrm{orb}}$. Fig. $1 \mathrm{~b}$ shows $\mathrm{M}_{\mathrm{BH}} / \mathrm{t}_{\mathrm{orb}}$ as a function of $\sigma$, where the filled circles are the mass accretion rate onto the sink particle per orbital time for the different runs, and the solid line is $<\dot{M}>\propto \sigma^{3}$. Thus, the condition $<\dot{M}>\propto \sigma^{3}$ is also satisfied in our simulations.

Finally, we plot the final MBH mass for the six different runs in Fig. 2. In each run, the host spheroid has a different mass and therefore a different total dynamical mass $\left(\mathrm{M}_{\text {star }}+\mathrm{M}_{\text {gas }}\right)$ enclosed within the initial disk. Fig. 2 shows a clear correlation between the $\mathrm{MBH}$ mass and the host spheroid mass. The solid line is $\mathrm{M}_{\mathrm{bh}} \propto \mathrm{M}_{\text {tot }}$ and the rms scatter is 0.104 dex (considerably less than in the ' $\mathrm{M}_{\mathrm{BH}}-\mathrm{M}_{\text {virial }}$ ' relation).

We find in Fig. 2 small amounts of scatter $(\mathrm{rms} \approx 0.1$ dex) compared to the scatter observed in the ' $\mathrm{M}_{\mathrm{BH}}-\mathrm{M}_{\mathrm{virial}}$ ' relation $(\mathrm{rms} \approx 0.25 \mathrm{dex}$; Marconi \& Hunt 2003). However, we think that the small amounts of scatter is expected given that our simulations are still idealized, with only one parameter varied and that only tries to reveal the physical process (fueling) responsible of the ' $\mathrm{M}_{\mathrm{BH}}-\mathrm{M}_{\mathrm{v} i r i a l}$ ' relation. Many other processes that are not taken into account in this work, such the number of major mergers (growth episodes) that happened in the history of a given spheroid, the feedback from star formation and possibly from an AGN, the fraction of the mass fed that ends up inside the event horizon of the $\mathrm{MBH}$, within other processes will contribute to increase the scatter in the relation.
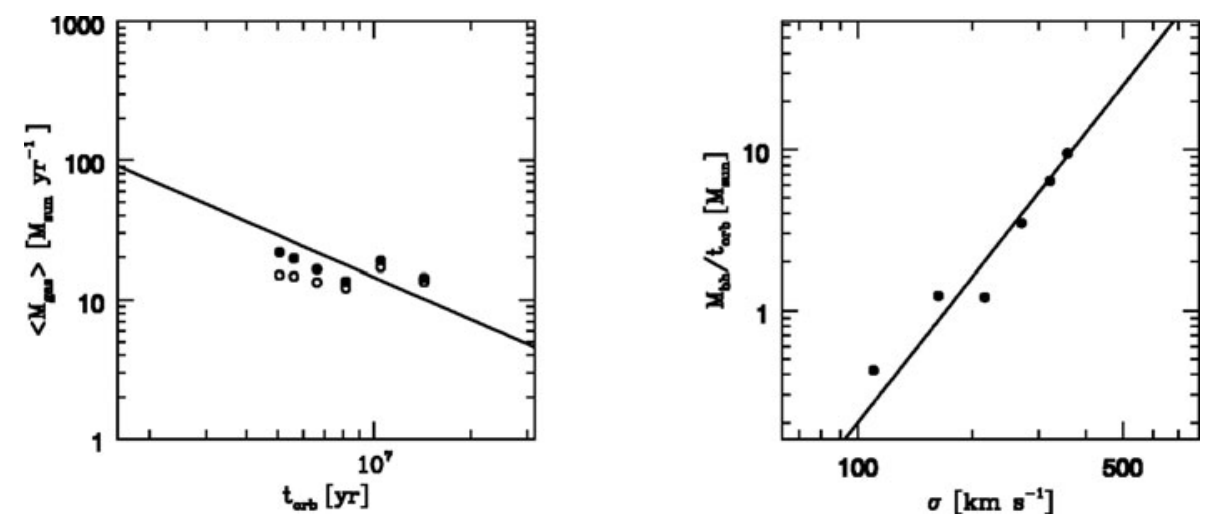

Figure 1. (a) Average gas depletion rate $<\dot{\mathrm{M}}_{\text {gas }}>$ as a function of the orbital time, for the six different runs. The open circles are the gas depletion rate due to only star formation, the filled circles are the gas depletion rate due to both star formation and accretion onto the $\mathrm{MBH}$. The solid line corresponds to $<\dot{\mathrm{M}}_{\text {gas }}>\propto \mathrm{t}_{\text {orb }}^{-1}$ (b) Total mass accreted by the MBH per orbital time (filled circles) plotted against the stellar velocity dispertion $\sigma$, for the different runs. The solid line corresponds to $\mathrm{M}_{\mathrm{BH}} / \mathrm{t}_{\mathrm{orb}} \propto \sigma^{3}$. 


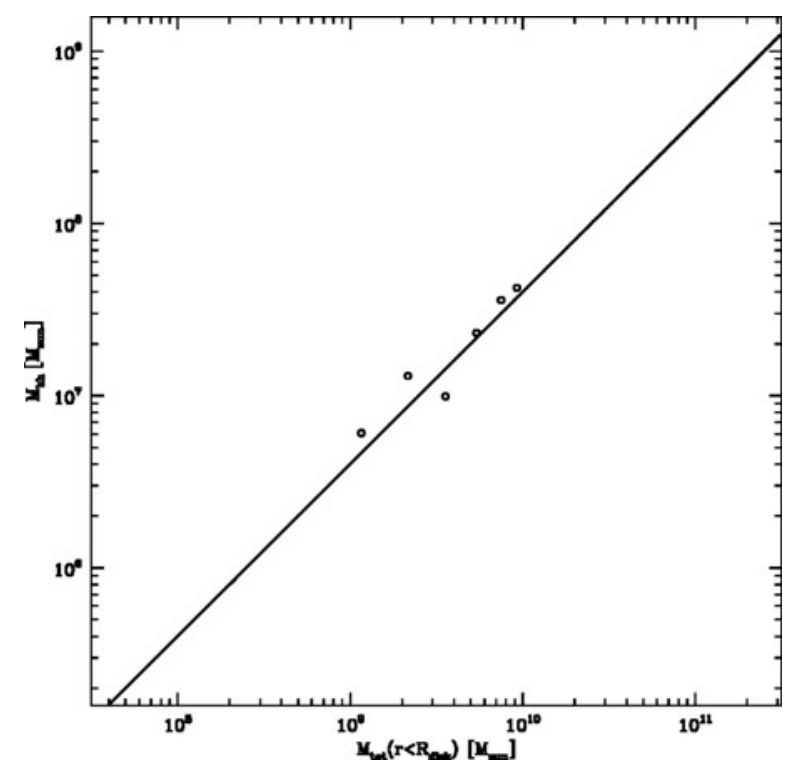

Figure 2. Final MBH mass plotted against the total dynamical mass $\left(M_{\text {gas }}+M_{\text {star }}\right)$ enclosed within the initial disk, for the six different runs (circles). The solid line corresponds to $\mathrm{M}_{\mathrm{BH}} \propto \mathrm{M}_{\mathrm{tot}}$.

\section{Conclusions}

We study sub-pc resolution AMR simulations of large-scale gas fueling, which is mainly depleted by star formation, onto a $\mathrm{MBH}$ with its accretion disk. We find that the central object naturally satisfy the ' $\mathrm{M}_{\mathrm{BH}}-\mathrm{M}_{\mathrm{virial}}$ ' relation. See Escala (2007) for more details about this work.

\section{References}

Barnes, J. E. 2002, MNRAS, 333, 481

Bryan, G. L. \& Norman, M. L. 1997, astro-ph/9710187v1

Cen, R. \& Ostriker, J. P. 1992, ApJ, 399L, 113

Dotti, M., Colpi, M., \& Haardt, F. 2006, MNRAS, 367, 103

Escala, A., et al. 2004, ApJ, 607, 765

Escala, A., et al. 2005, ApJ, 630, 152

Escala, A. 2006, ApJ, 648, L13

Escala, A. 2007, astroph/0705.4457

Ferrarese, L. \& Merritt, D. 2000, ApJ, 539, 9

Gebhardt, K., et al. 2000, ApJ, 539, 13

Haring, N. \& Rix, H. W. 2004, ApJ, 604L, 89

Kazantzidis, S., et al. 2005, ApJ, 623L, 67

Kennicutt, R. C. 1998, ApJ, 498, 541

Kormendy, J., \& Richstone, D., ARA\&A, 33, 581

Mayer, L. et al. 2007, astroph/0706.1562

Marconi, A. \& Hunt, L. K. 2003, ApJ, 589L, 21

Shlosman, I., Begelman, M., \& Frank, J. 1990, Nature, 345, 679 